\title{
Tazarotene-induced gene 2 is associated with poor survival in non-small cell lung cancer
}

\author{
QI CAI $^{1^{*}}$, ZHONGWEI HUANG $^{2 *}$, LEI $^{2}{ }^{2}$, TING WANG $^{2}$, YANBO SHEN $^{2}$ and JIAN'AN HUANG ${ }^{1}$ \\ ${ }^{1}$ Department of Respiratory and Critical Care Medicine, First Affiliated Hospital of Soochow University, \\ Suzhou, Jiangsu 215006; ${ }^{2}$ Department of Emergency Medicine, \\ Affiliated Hospital of Nantong University, Nantong, Jiangsu 226001, P.R. China
}

Received March 24, 2015; Accepted April 12, 2016

DOI: $10.3892 / \mathrm{ol} .2016 .5025$

\begin{abstract}
The aim of the present study was to investigate the expression of tazarotene-induced gene 2 (TIG2) and evaluate the clinicopathological variables and prognostic value for non-small cell lung cancer (NSCLC) patients. Reverse transcription-polymerase chain reaction and western blotting were utilized to detect TIG2 expression in NSCLC specimens and adjacent noncancerous tissue. Furthermore, the present study investigated the protein expression and the clinicopathological significance of TIG2 in 98 paraffin-embedded NSCLC samples by using immunohistochemistry. The results of the present study demonstrated that the expression of TIG 2 mRNA $(\mathrm{P}=0.003)$ and protein $(\mathrm{P}=0.0024)$ was significantly reduced in NSCLC compared with corresponding noncancerous tissue. TIG2 protein expression in NSCLC was significantly associated with lymph node metastasis $(\mathrm{P}=0.006)$, Tumor-Node-Metastasis stage $(\mathrm{P}=0.021)$ and degree of differentiation $(\mathrm{P}=0.025)$. The Kaplan-Meier method and log-rank test revealed that high TIG2 expression was significantly associated with increased survival of NSCLC patients $(\mathrm{P}=0.003)$. Multivariate analysis revealed that TIG2 expression was an independent prognostic factor of the overall survival of NSCLC patients. Decreased expression of TIG2 may be useful as a biomarker for poor prognosis in NSCLC carcinogenesis and may act as a target for gene therapy for the treatment of NSCLC patients.
\end{abstract}

Correspondence to: Professor Jian'an Huang, Department of Respiratory and Critical Care Medicine, First Affiliated Hospital of Soochow University, 188 Shizi Street, Suzhou, Jiangsu 215006, P.R. China

E-mail: jiananhuang1@163.com

${ }^{*}$ Contributed equally

Key words: tazarotene-induced gene 2, non-small cell lung cancer, prognosis

\section{Introduction}

Lung cancer is a malignant tumor with a high incidence and mortality rate; it is currently the number one cause of cancer-associated mortality worldwide, with $\sim 1.4$ million deaths annually (1). Lung cancer is diagnosed during its advanced stages in the majority of patients, which is the primary reason underlying the high mortality rate associated with this disease (2). Non-small cell lung cancer (NSCLC), including adenocarcinoma, squamous cell carcinoma, large cell carcinoma and bronchioloalveolar carcinoma, accounts for $\sim 85 \%$ of all lung cancer cases (3). Despite numerous available adjuvant therapies, the overall five-year survival rate remains poor for lung cancer patients (4), and the most frequent causes of cancer-associated mortality are the occurrence of local invasion or distant metastasis, rather than the primary tumors (5). Therefore, investigating novel markers and the molecular mechanisms that are involved in NSCLC is of great importance for improving the diagnosis and treatment of NSCLC.

Retinoids regulate the growth, differentiation, and apoptosis of healthy cells during embryonic development and of premalignant and malignant cells during carcinogenesis (6). It has been proposed that nuclear retinoid receptors may mediate these effects via the regulation of gene transcription (7). The regulation of gene transcription is controlled by the activation of two types of nuclear receptors: Retinoic acid receptors (RAR $\alpha, \operatorname{RAR} \beta$ and $\operatorname{RAR} \gamma$ ) and retinoid $X$ receptors (RXR $\alpha, \operatorname{RXR} \beta$ and $\mathrm{RXR} \gamma)(8)$. Tazarotene, a synthetic retinoid that binds $\operatorname{RAR} \beta$ and $\operatorname{RAR} \gamma$, is utilized in the treatment of psoriasis (9). Tazarotene upregulates three genes: Tazarotene-induced gene (TIG)1, TIG2 and TIG3, which may lead to an anti-proliferative effect (10). Initially, TIG2 was identified in a subtraction hybridization assay, which observed upregulated genes of human skin raft cultures by treatment with the anti-psoriatic retinoid drug tazarotene (11). TIG2 is expressed in the skin, pancreas, liver, spleen, prostate, ovary, small intestine and colon (12). A small amount of evidence has demonstrated that TIG2 may be a tumor suppressor gene (13); however, an association between TIG2 expression, clinicopathological characteristics and the prognosis in NSCLC remains to be elucidated.

The present study sought to demonstrate TIG2 expression in patients with NSCLC using reverse transcription-quantitative 
polymerase chain reaction (RT-qPCR), western blotting and immunohistochemical analysis. Subsequently, the present study investigated the association between TIG2 expression and clinicopathological parameters to establish whether TIG2 may serve as a novel prognostic biomarker in NSCLC patients.

\section{Materials and methods}

Patients and specimens. Relevant clinicopathological data from 98 lung cancer patients who underwent surgical resection at the Affiliated Hospital of Nantong University (Nantong, China) between January 2006 and December 2008 were collected and retrospectively analyzed. These human NSCLC tissue samples had been formalin-fixed, paraffin-embedded and histopathologically diagnosed. Additionally, for RT-qPCR and western blot analysis, the present study collected 32 paired fresh NSCLC tumor tissue samples and corresponding adjacent noncancerous tissue samples from patients who underwent surgery between January 2013 and December 2014. None of the patients received preoperative chemotherapy or radiation therapy. A follow-up evaluation was performed every 2 months for the initial 2 years following the operation, every 3 months in the third year and every 6 months after this. Informed consent was obtained from all patients, and the study was approved by the Institutional Hospital Ethics Committee.

$R T$ - $q P C R$. Total RNA was extracted from tissues using TRIzol reagent according to the manufacturer's protocol (Invitrogen; Thermo Fisher Scientific, Inc., Waltham, MA, USA). RNA was reverse transcribed using an Omniscript Reverse Transcription kit (Qiagen, Inc., Valencia, CA, USA) according to the manufacturer's protocol. The PCR amplification was performed at $96^{\circ} \mathrm{C}$ for $2 \mathrm{~min}$, followed by 40 cycles of $96^{\circ} \mathrm{C}$ for $15 \mathrm{sec}$ and $60^{\circ} \mathrm{C}$ for $1 \mathrm{~min}$, on a Mastercycler ep realplex (Eppendorf, Hamburg, Germany) with $1.0 \mu \mathrm{l}$ of cDNA and 2X Fast EvaGreen $^{\text {TM }}$ qPCR Master Mix (Biotium, Inc., Fremont, CA, USA). The expression level of each candidate gene was internally normalized against that of glyceraldehyde-3-phosphate dehydrogenase (GAPDH; internal control). The relative quantitative value was expressed by the $2^{-\Delta \Delta \mathrm{Cq}}$ method (14). Each experiment was performed in triplicate. The primer sequences used were as follows: TIG2 forward, AATGGGAGGAAA CGGAAATGC and reverse, GCGAACTGTCCAGGGAAG TAGAA; and GAPDH forward, 5'-AACTTCCGTTGCTGC CAT-3' and reverse, 5'-TTTCTTCCACAGGGCTTTG-3'. Negative controls (no cDNA) and RT controls (no RT) were performed.

Western blot analysis. The frozen tissue samples from patients with NSCLC, including the tumor tissues and adjacent noncancerous tissues, were homogenized in radioimmunoprecipitation assay lysis buffer (Beyotime Institute of Biotechnology, Haimen, China), and the lysates were cleared by centrifugation $(13,523 \mathrm{x} \mathrm{g})$ at $4^{\circ} \mathrm{C}$ for $15 \mathrm{~min}$. Protein samples $(20 \mu \mathrm{g})$ were separated by $10 \%$ SDS-PAGE and subsequently transferred to nitrocellulose membranes. The membranes were blocked with 5\% non-fat dry milk in Tris-Buffered Saline and Tween 20 (TBST) buffer for $1 \mathrm{~h}$ at room temperature and incubated with a monoclonal mouse anti-human TIG2 antibody (dilution, 1:500; cat. no. sc-373797; Santa Cruz Biotechnology,
Inc., Dallas, TX, USA) at $4^{\circ} \mathrm{C}$ overnight. Following washing three times for 5 min each in TBST, the membranes were incubated with a horseradish peroxidase (HRP)-conjugated goat anti-mouse secondary antibody (dilution, 1:5,000; cat. no. L3032-2; Signalway Antibody, Nanjing, China) for $2 \mathrm{~h}$ at room temperature. Following washing 3 times for $5 \mathrm{~min}$ with TBST, protein bands were visualized by scanning with an Odyssey ${ }^{\circledR}$ CLx Infrared Imaging System (LI-COR Biosciences, Lincoln, NE, USA). The acquired band intensity was measured by densitometry using Quantity One 4.62 software (Bio-Rad Laboratories, Inc., Hercules, CA, USA). The protein levels were normalized to that of GAPDH.

Immunohistochemistry. Paraffin-embedded sections of surgically resected specimens were routinely dewaxed through a series of xylene and were rehydrated with graded ethanol. Sections were pretreated by high-pressure mediated antigen retrieval buffer (Abcam, Cambridge, UK) with $0.01 \mathrm{M}$ sodium citrate-hydrochloric acid $(\mathrm{pH}=6.0)$ at $100 \mathrm{Kpa}$ for $5 \mathrm{~min}$. Slides were blocked in $3 \% \mathrm{H}_{2} \mathrm{O}_{2}$ for $10 \mathrm{~min}$. The sections were incubated with monoclonal mouse anti-human TIG2 antibody (dilution, 1:100) overnight at $4^{\circ} \mathrm{C}$. After washing three times for 5 min each with TBST, the sections were incubated with HRP-conjugated goat anti-mouse secondary antibody (dilution, $1: 5,000$ ) at $4^{\circ} \mathrm{C}$ overnight, and then visualized with 3,3'-diaminobenzidine solution. All of the slides were counterstained with hematoxylin. Immunostaining of TIG2 was scored following a semiquantitative scale by evaluating representative tumor areas, intensity and percentage of cells. Staining was classified as positive or negative, and was scored as follows: Intensity $(0$, negative; 1 , weak; 2 , moderate; and 3 , strong); and percentage of positive tumor cells $(0,<5 \%$; $1,<25 \% ; 2,26-50 \% ; 3,51-75 \%$; and 4, >75\%). The scores for intensity and percentage of positive tumor cells of each sample were multiplied (range 0-12), and the total expression of TIG2 was determined as: Low (negative, <1; weak, 1-4); or high (moderate, 5-8; strong, 9-12). All stained sections were evaluated and scored individually by two pathologists with no prior knowledge of the clinicopathological consequences of the patients.

Statistical analysis. Statistical analyses were performed using SPSS version 18.0 (SPSS, Inc., Chicago, IL, USA). Correlation between TIG2 expression and clinicopathological characteristics was evaluated using the $\chi^{2}$ test. The 5-year survival rates following tumor resection were calculated using the Kaplan-Meier method, and differences in overall survival curves were analyzed using the log-rank test. Multivariate analysis was performed using the Cox proportional hazards regression model on several prognostic factors. $\mathrm{P}<0.05$ was considered to indicate a statistically significant difference.

\section{Results}

Expression levels of TIG2 in NSCLC. Western blot analysis revealed that the expression level of TIG2 was markedly decreased in 16/20 NSCLC tissues compared with the corresponding adjacent noncancerous tissues. A total of 7 representative western blot analysis results are presented in Fig. 1A. The average TIG2 protein level in 20 NSCLC tissues 


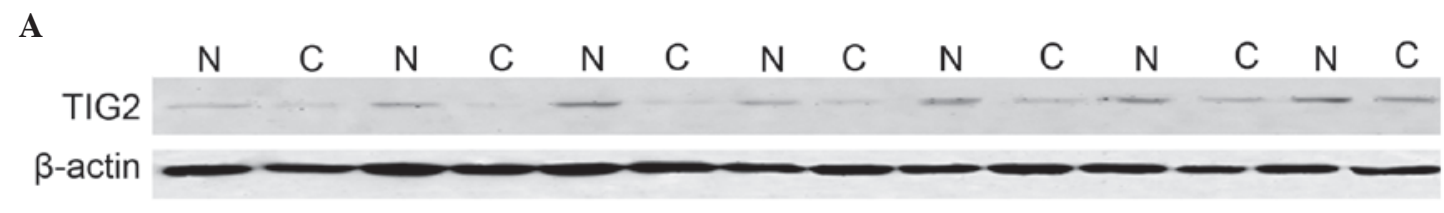

B

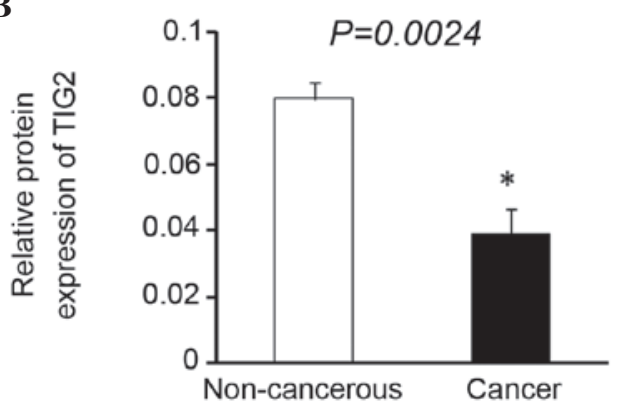

C

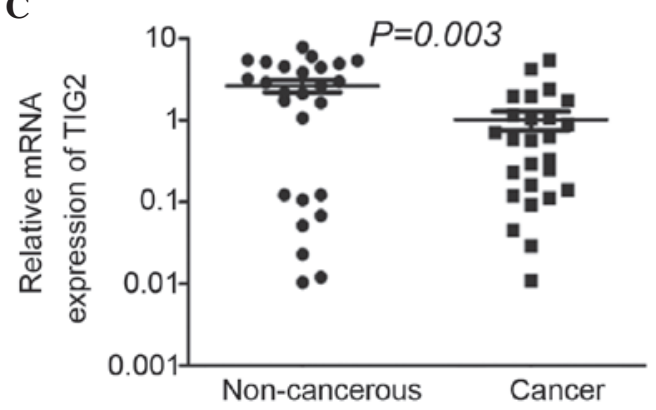

Figure 1. Expression of TIG2 protein and mRNA in NSCLC tissues and adjacent noncancerous tissues was evaluated by western blotting and reverse transcription-quantitative polymerase chain reaction. (A) Western blot analysis of TIG2 protein expressed in 7 paired representative NSCLC tissues and matched adjacent noncancerous tissues. $\beta$-actin was used as a control for equal loading. (B) Relative TIG2 protein expression levels were markedly decreased in $16 / 20$ NSCLC tissues compared with the corresponding adjacent noncancerous tissues $(\mathrm{P}=0.0024)$. $\mathrm{P}<0.05$. (C) Normalized to glyceraldehyde-3-phosphate dehydrogenase mRNA levels, the TIG 2 mRNA level in NSCLC tissues was significantly lower than that in corresponding noncancerous tissues ( $\mathrm{P}=0.003)$. TIG2, tazarotene-induced gene 2; NSCLC, non-small cell lung cancer; C, cancerous tissue; N, noncancerous tissue.

was significantly reduced compared with that of TIG2 in adjacent normal tissues ( $\mathrm{P}=0.0024$; Fig. 1B). In addition, RT-qPCR was performed to detect the expression levels of TIG2. The results of the present study demonstrated that TIG2 mRNA expression was downregulated in 18/26 NSCLC samples compared with corresponding adjacent noncancerous tissues. The mean expression value of mRNA in NSCLC tissues was significantly reduced compared with the value in paired adjacent noncancerous tissues ( $\mathrm{P}=0.003$; Fig. $1 \mathrm{C})$.

To additionally investigate the expression of TIG2 protein in cancer and corresponding adjacent noncancerous tissues, immunohistochemical analysis was performed on NSCLC specimens. TIG2 expression was detected at various levels, primarily in the cytoplasm of cells. High TIG2 expression was detected in $41.83 \%$ (41/98) of NSCLC samples, whereas the remaining $58.17 \%$ (57/98) displayed low TIG2 expression (Fig. 2). The above results indicated that TIG2 may have a tumor suppressor role in NSCLC.

Correlation of clinicopathological features and TIG2 expression. To additionally investigate the significance of TIG2 expression in NSCLC, the present study evaluated the correlation of TIG2 expression with clinicopathological data. The expression levels of TIG2 in NSCLC tissues were categorized as low or high depending on the mean score. As shown in Table I, the $\chi^{2}$ analysis revealed that the TIG2 level was associated with lymph node metastasis $(\mathrm{P}=0.006)$, Tumor-Node-Metastasis (TNM) stage $(\mathrm{P}=0.021)$ and differentiation $(\mathrm{P}=0.025)$. However, there was no significant correlation between TIG2 expression and other clinicopathological features, including age, gender, tumor size, smoking history, histological classification or distant metastasis ( $\mathrm{P}>0.05$ in all cases).

Downregulated expression level of TIG2 predicts poor prognosis in NSCLC patients. To investigate the prognostic value of TIG2 expression in NSCLC patients, survival analysis was performed to assess the effect of TIG2 on survival time of NSCLC patients. Kaplan-Meier curves for overall survival are presented in Fig. 3. Log-rank test was utilized for statistical analysis. Patients with low TIG2 expression possessed shorter overall survival times compared with those with high TIG2 expression $(\mathrm{P}=0.003)$.

Furthermore, multivariate analysis revealed that TIG2 expression, TNM stage and differentiation were independent prognostic markers for NSCLC (Table II; $\mathrm{P}<0.05$ in all cases). In conclusion, the results of the present study suggested that patients with lower expression of TIG2 in NSCLC tissues demonstrated poorer overall survival compared with patients with higher expression, providing evidence that decreased expression of TIG2 in NSCLC may facilitate increased malignancy and a poorer prognostic phenotype.

\section{Discussion}

Tazarotene upregulates the expression of TIG1 TIG2, and TIG3 (10). TIG1 may function as a cell adhesion molecule, whose expression on the cell surface may lead to improved cell-cell contact and a reduction in proliferation, which may additionally have a tumor suppressive role by acting as a retinoid mediator of the cells (15). TIG3, also known as a class II tumor suppressor via interaction with retinoids, has been demonstrated to inhibit cellular proliferation when expressed and is associated with retinoid responsiveness in malignant cell lines in vitro (16). TIG2, also known as chemerin, has been characterized as a chemotactic agent and was identified as the ligand for an orphan $\mathrm{G}$ protein-coupled receptor, ChemR23, which is expressed by immature dendritic cells and macrophages (17). Previously, it was reported that chemerin recruits ChemR23 receptor (chemerinR)-expressing dendritic cells and macrophages, suggesting a regulatory function in the development of the immune response (18). A high expression level of chemerinR transcript was additionally detected in the 
Table I. Correlation of TIG2 expression in tumor tissues with clinicopathological characteristics in non-small cell lung cancer patients .

\begin{tabular}{|c|c|c|c|c|}
\hline \multirow[b]{2}{*}{ Characteristic } & \multirow[b]{2}{*}{ Total, $\mathrm{n}$} & \multicolumn{2}{|c|}{ TIG2 expression } & \multirow[b]{2}{*}{ P-value } \\
\hline & & $\operatorname{High}(n=41)$ & Low $(n=57)$ & \\
\hline Age, years & & & & 0.258 \\
\hline$<60$ & 46 & 22 & 24 & \\
\hline$\geq 60$ & 52 & 19 & 33 & \\
\hline Gender & & & & 0.182 \\
\hline Male & 72 & 33 & 39 & \\
\hline Female & 26 & 8 & 18 & \\
\hline Tumor size, cm & & & & 0.342 \\
\hline$<5$ & 65 & 25 & 40 & \\
\hline$\geq 5$ & 33 & 16 & 17 & \\
\hline Smoking history & & & & 0.104 \\
\hline Smoker & 64 & 23 & 41 & \\
\hline Non-smoker & 34 & 18 & 16 & \\
\hline Lymph node metastasis & & & & $0.006^{\mathrm{a}}$ \\
\hline Negative & 61 & 32 & 29 & \\
\hline Positive & 37 & 9 & 28 & \\
\hline Histological classification & & & & 0.657 \\
\hline Squamous cell carcinoma & 39 & 18 & 21 & \\
\hline Adenocarcinoma & 55 & 22 & 33 & \\
\hline Other & 4 & 1 & 3 & \\
\hline Tumor-Node-Metastasis stage & & & & $0.021^{\mathrm{a}}$ \\
\hline $\mathrm{I} / \mathrm{II}$ & 69 & 34 & 35 & \\
\hline III/IV & 29 & 7 & 22 & \\
\hline Differentiation & & & & $0.025^{\mathrm{a}}$ \\
\hline Well/moderate & 42 & 23 & 19 & \\
\hline Poor & 56 & 18 & 38 & \\
\hline Distant metastasis & & & & 0.211 \\
\hline M0 & 89 & 39 & 50 & \\
\hline M1 & 9 & 2 & 7 & \\
\hline
\end{tabular}

${ }^{a} \mathrm{P}<0.05$ was considered to indicate a statistically significant difference. TIG2, tazarotene-induced gene 2; M0, no distant metastasis; M1, distant metastasis.

Table II. Multivariate analysis of prognostic factors in non-small cell lung cancer for overall survival by Cox regression model.

\begin{tabular}{lrr}
\hline & & \multicolumn{2}{c}{ Multivariate analysis } \\
\cline { 2 - 3 } Characteristic & HR & $95 \%$ CI \\
\hline Tumor size, cm ( $\geq 5$ vs. <5) & 1.242 & $0.346-2.864$ \\
Histology (squamous cell carcinoma vs. adenocarcinoma) & 1.051 & $0.672-2.162$ \\
Smoking history (smoker vs. non-smoker) & 1.429 & $0.799-2.786$ \\
Lymph node metastasis (positive vs. negative) & 0.894 & $0.562-1.876$ \\
Tumor-Node-Metastasis stage (III-IV vs. I-II) & 2.397 & $1.426-3.048$ \\
Differentiation (Well/moderate vs. poor) & 1.873 & $1.270-3.392$ \\
Distant metastasis (M1 vs. M0) & 2.674 & $1.632-3.893$ \\
Tazarotene-induced gene 2 expression (high vs. low) & 2.225 & $1.332-4.291$ \\
\hline
\end{tabular}

${ }^{\mathrm{a}} \mathrm{P}<0.05$ was considered to indicate a statistically significant difference. HR, hazard ratio; CI, confidence interval; M0, no distant metastasis; M1, distant metastasis. 
A

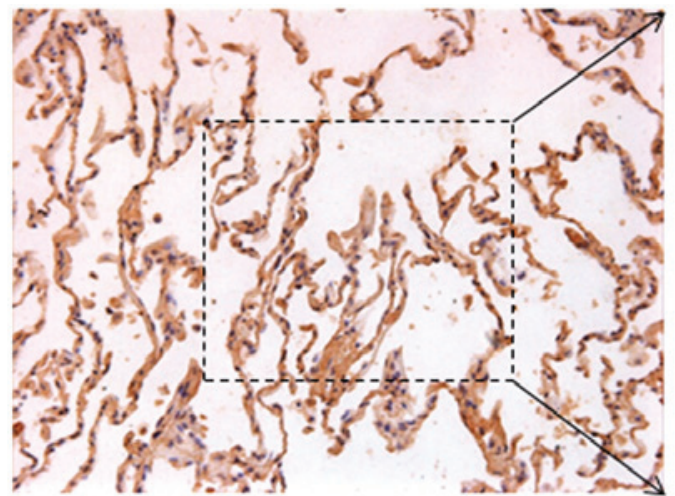

C

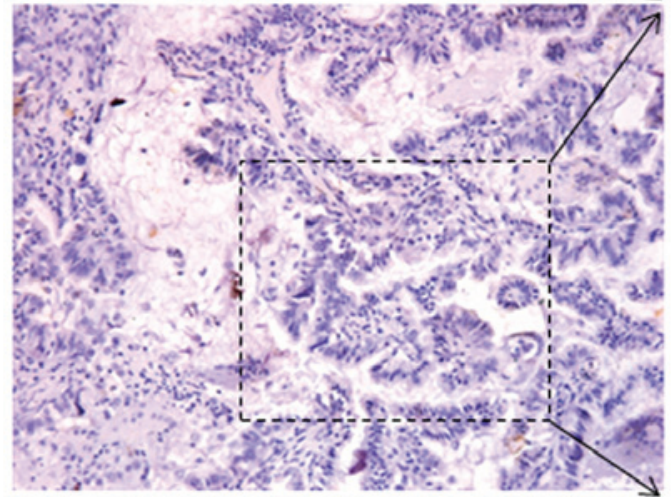

$\mathbf{E}$

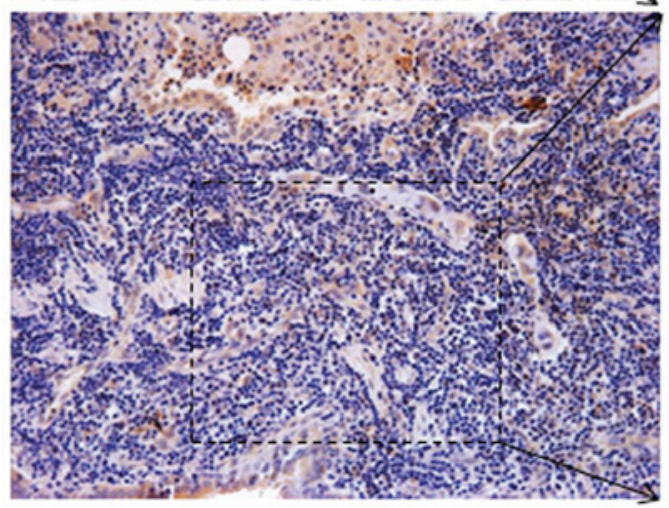

B

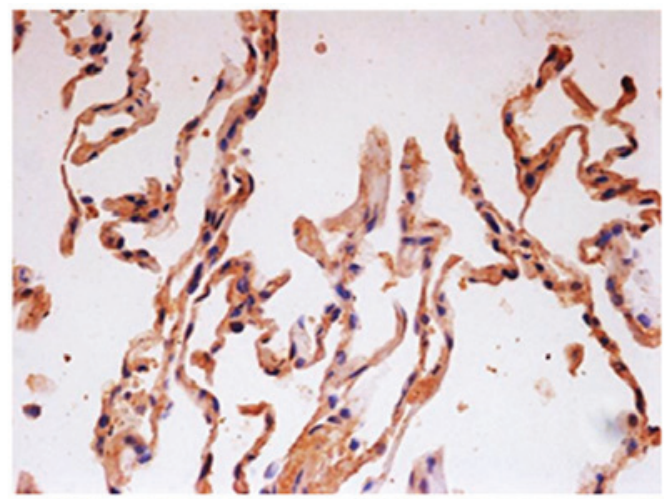

D

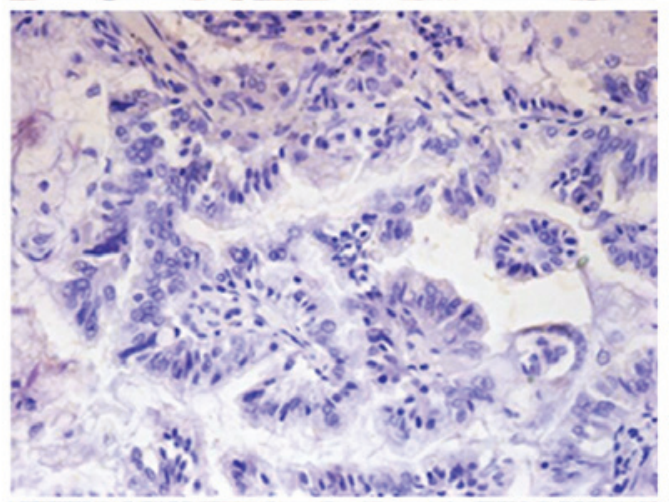

$F$

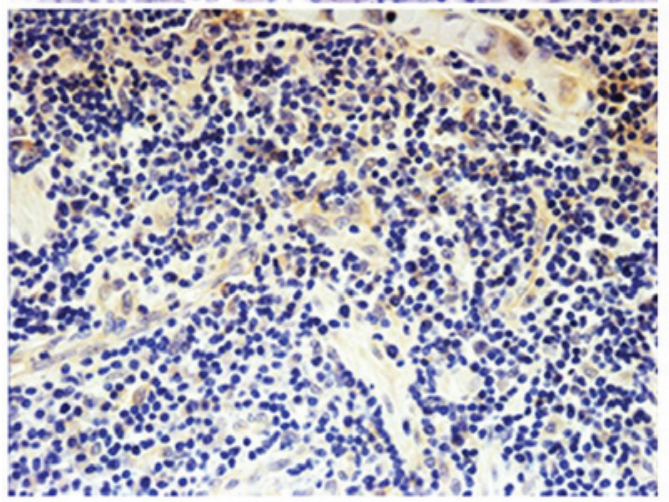

Figure 2. Representative images of TIG2 protein expression in non-small cell lung cancer and adjacent noncancerous tissues. (A and B) Strong cytoplasmic staining of TIG2 in tumor adjacent noncancerous tissues. (C and D) Weak cytoplasmic staining of TIG2 in lung adenocarcinoma tissues. (E and F) Weak cytoplasmic staining of TIG2 in lung squamous cell carcinoma tissues. Magnification, x200 in left panel and x400 in right panel. TIG2, tazarotene-induced gene 2.

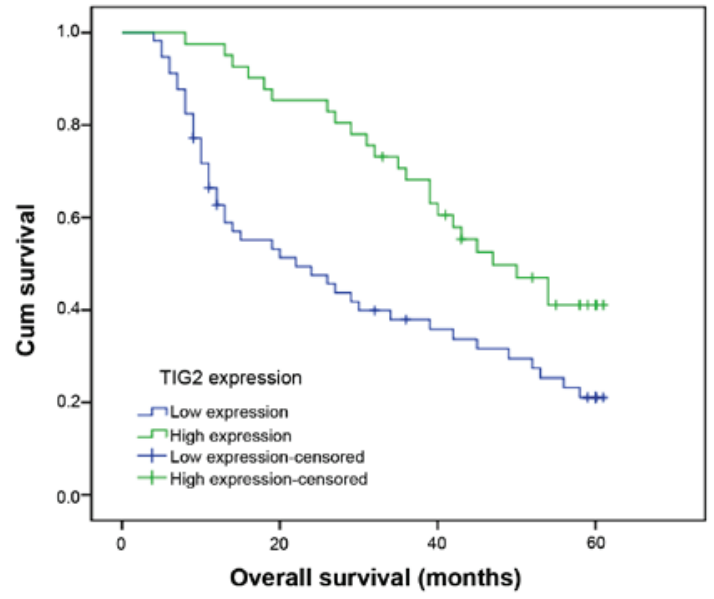

Figure 3. Kaplan-Meier curves of the overall survival of 98 non-small cell lung carcinoma patients. The overall survival rate in patients with low TIG2 expression was significantly reduced compared with that in patients with high TIG2 expression $\left(\chi^{2}=8.776 ; \mathrm{P}=0.003\right)$. TIG2, tazarotene-induced gene 2 Cum, cumulative. spleen, lymph nodes and lung. Simultaneous expression of receptor and chemerin mRNA transcript was observed in the spleen, lymph node and lung (17). However, to the best of our knowledge, the prognostic significance of TIG2 in NSCLC has not been investigated. In the present study, the expression of TIG2 was evaluated in NSCLC by RT-qPCR, western blotting and immunohistochemistry. The present study additionally analyzed the clinicopathological and prognostic significance of TIG2 in a large number of human samples. It was demonstrated that TIG2 was expressed at reduced levels in terms of mRNA and protein in NSCLC tissues compared with corresponding adjacent noncancerous tissues. Immunohistochemical staining analysis additionally demonstrated that TIG2 expression was decreased in 57/98 NSCLC tissues. However, the mechanism of downregulation of TIG2 expression during the occurrence and development of NSCLC remains to be elucidated, and requires additional investigation in future studies.

Furthermore, the present study observed that reduced expression of TIG2 was significantly associated with lymph 
node metastasis, TNM stage and level of differentiation, suggesting that aberrant expression of TIG2 may participate in NSCLC tumor development and progression. In Kaplan-Meier curve analysis of the overall survival of 98 NSCLC patients, the overall survival rate in patients with low TIG2 expression was significantly reduced compared with that in patients with high TIG2 expression. In addition, Cox multivariate analysis revealed that TIG2 expression was an independent prognostic factor for overall survival in NSCLC tissues. The results of the present study suggested that TIG 2 expression may be a potential molecular marker for predicting outcomes in NSCLC patients.

In conclusion, the present study demonstrated that the expression of TIG2 was significantly decreased in NSCLC tissues. Downregulated expression of TIG2 was significantly associated with tumor progression and decreased survival in patients with NSCLC, indicating that TIG2 may act as a novel prognostic marker for the diagnosis and treatment of NSCLC patients.

\section{References}

1. Jemal A, Center MM, DeSantis C and Ward EM: Global patterns of cancer incidence and mortality rates and trends. Cancer Epidemiol Biomarkers Prev 19: 1893-1907, 2010.

2. Heighway J and Betticher DC: Lung tumors: an overview. Atlas Genet Cytogenet Oncol Haematol 8: 139-141, 2004

3. Ramalingam SS, Owonikoko TK and Khuri FR: Lung cancer: New biological insights and recent therapeutic advances. CA Cancer J Clin 61: 91-112, 2011.

4. Siegel R, DeSantis C, Virgo K, Stein K, Mariotto A, Smith T, Cooper D, Gansler T, Lerro C, Fedewa S, et al: Cancer treatment and survivorship statistics, 2012. CA Cancer J Clin 62: 220-241, 2012.

5. Morgensztern D, Ng SH, Gao F and Govindan R: Trends in stage distribution for patients with non-small cell lung cancer: A National Cancer Database survey. J Thorac Oncol 5: 29-33, 2010.

6. Seewaldt VL, Johnson BS, Parker MB, Collins SJ and Swisshelm K: Expression of retinoic acid receptor beta mediates retinoic acid-induced growth arrest and apoptosis in breast cancer cells. Cell Growth Differ 6: 1077-1088, 1995.
7. Huang P, Chandra V and Rastinejad F: Structural overview of the nuclear receptor superfamily: Insights into physiology and therapeutics. Annu Rev Physiol 72: 247-272, 2010.

8. Boehm MF, Heyman RA, Patel S, Stein RB and Nagpal S: Section Review: Retinoids: Biological function and use in the treatment of dermatological diseases: Pulmonary-allergy, dermatological, gastrointestinal \& arthritis. Expert Opin Investig Drugs 4: 593-612, 2008

9. Chandraratna RA: Tazarotene: The first receptor-selective topical retinoid for the treatment of psoriasis. J Am Acad Dermatol 37 (Suppl): S12-S17, 1997.

10. Duvic M, Nagpal S, Asano AT and Chandraratna RA: Molecular mechanisms of tazarotene action in psoriasis. J Am Acad Dermatol 37 (Suppl): S18-S24, 1997.

11. Nagpal S, Patel S, Jacobe H, DiSepio D, Ghosn C, Malhotra M, Teng M, Duvic M and Chandraratna RA: Tazarotene-induced gene 2 (TIG2), a novel retinoid-responsive gene in skin. J Invest Dermatol 109: 91-95, 1997.

12. Adams AE, Abu-Amer Y, Chappel J, Stueckle S, Ross FP, Teitelbaum SL and Suva LJ: 1,25 dihydroxyvitamin D3 and dexamethasone induce the cyclooxygenase 1 gene in osteoclast-supporting stromal cells. J Cell Biochem 74: 587-595, 1999.

13. Zheng Y, Luo S, Wang G, Peng Z, Zeng W, Tan S, Xi Y and Fan J: Downregulation of tazarotene induced gene-2 (TIG2) in skin squamous cell carcinoma. Eur J Dermatol 18: 638-641, 2008.

14. Livak and Schmittgen: Analysis of relative gene expression data using real-time quantitative PCR and the $2-\Delta \Delta \mathrm{Ct}$ method. Methods 25: 402-408, 2001.

15. Jing C, El-Ghany MA, Beesley C, Foster CS, Rudland PS, Smith P and Ke Y: Tazarotene-induced gene 1 (TIG1) expression in prostate carcinomas and its relationship to tumorigenicity. J Natl Cancer Inst 94: 482-490, 2002.

16. DiSepio D, Ghosn C, Eckert RL, Deucher A, Robinson N, Duvic M, Chandraratna RA and Nagpal S: Identification and characterization of a retinoid-induced class II tumor suppressor/growth regulatory gene. Proc Natl Acad Sci USA 95: 14811-14815, 1998.

17. Wittamer V, Franssen JD, Vulcano M, Mirjolet JF, Le Poul E, Migeotte I, Brézillon S, Tyldesley R, Blanpain C, Detheux M, et al: Specific recruitment of antigen-presenting cells by chemerin, a novel processed ligand from human inflammatory fluids. J Exp Med 198: 977-985, 2003.

18. Luangsay S, Wittamer V, Bondue B, De Henau O, Rouger L, Brait M, Franssen JD, de Nadai P, Huaux F and Parmentier M: Mouse ChemR23 is expressed in dendritic cell subsets and macrophages, and mediates an anti-inflammatory activity of chemerin in a lung disease model. J Immunol 183: 6489-6499, 2009. 